\title{
THE TREE OF IRREDUCIBLE NUMERICAL SEMIGROUPS WITH FIXED FROBENIUS NUMBER
}

\author{
V. BLANCO AND J.C. ROSALES \\ Departamento de Álgebra, Universidad de Granada \\ vblanco@ugr.es jrosales@ugr.es
}

\begin{abstract}
In this paper we present a procedure to build the set of irreducible numerical semigroups with a fixed Frobenius number. The construction gives us a rooted tree structure for this set. Furthermore, by using the notion of Kunz-coordinates vector we translate the problem of finding such a tree into the problem of manipulating $0-1$ vectors with as many component as the Frobenius number.
\end{abstract}

\section{INTRODUCTION}

A numerical semigroup is a subset $S$ of $\mathbb{N}$ (here $\mathbb{N}$ denotes the set of nonnegative integers) closed under addition, containing zero and such that $\mathbb{N} \backslash S$ is finite. The largest integer not belonging to $S$ is called the Frobenius number of $S$ and we denote it by $\mathrm{F}(S)$.

A numerical semigroup is irreducible if it cannot be expressed as an intersection of two numerical semigroups containing it properly. This notion was introduced in [8] where it is also proven that a numerical semigroup is irreducible if and only if $S$ is maximal (with respect to the inclusion ordering) in the set of numerical semigroups with Frobenius number $\mathrm{F}(S)$. From [2] and [4] it is also deduced in 8] that the family of irreducible numerical semigroups is the union of two families of numerical semigroups with special importance in this theory: symmetric and pseudo-symmetric numerical semigroups.

For a given positive integer $F$, we denote by $\mathcal{I}(F)$ the set of irreducible numerical semigroups with Frobenius number $F$. The main goal of this paper is to show how to build, iteratively, all the elements in $\mathcal{I}(F)$. Furthermore, such a construction will be represented as a rooted tree.

For the sake of simplicity of the procedure we use the notion of Kunz-coordinates vector, previously introduced in [3, to encode the numerical semigroups as $0-1$ vectors with as many coordinates as its Frobenius number. The equivalence between the numerical coordinate and its Kunz-coordinates vector allows us to translate the construction of the tree of irreducible numerical semigroups with fixed Frobenius number into an easy procedure by manipulating $0-1$ vectors.

The paper is organized as follows. We present the construction of the tree of numerical semigroups with fixed Frobenius number in Section 2 In Section 3 we give the notion of Kunz-coordinates vector of a numerical semigroups and translate the problem of building the tree into the problem of finding adequate $0-1$ 
vectors. It leads us to an efficient procedure to compute the set of irreducible numerical semigroups with Frobenius number $F$ by swapping elements in a vector with components in $\{0,1\}$.

\section{The tree of irReducible numerical semigroups With Frobenius NUMBER $F$}

If $A$ is a nonempty subset in $\mathbb{N}$, we denote by $\langle A\rangle$ the submonoid of $(\mathbb{N},+)$ generated by $A$, that is $\langle A\rangle=\left\{\lambda_{1} a_{1}+\cdots+\lambda_{n} a_{n}: n \in \mathbb{N} \backslash\{0\}, a_{1}, \ldots, a_{n} \in\right.$ $A$, and $\left.\lambda_{1}, \ldots, \lambda_{n} \in \mathbb{N}\right\}$. It is well-known that $\langle A\rangle$ is a numerical semigroup if and only if $\operatorname{gcd}(A)=1$. If $S$ is a numerical semigroup and $S=\langle A\rangle$, then we say that $A$ is a system of generators of $S$. In [9] it is shown that every numerical semigroup admits an unique minimal system of generators and that such a system is finite.

The following result has an immediate proof.

Lemma 1. Let $S$ be a numerical semigroup and $x$ a minimal generator of $S$. Then $S \backslash\{x\}$ is a numerical semigroups.

Let $S$ be a numerical semigroup. Following the notation in [6], we say that an integer $x \in \mathbb{Z} \backslash S$ is a pseudo-Frobenius number of $S$ if $x+s \in S$ for all $s \in S \backslash\{0\}$. We denote by $\operatorname{PF}(S)$ the set of pseudo-Frobenius numbers of $S$.

From lemmas 20 and 24 in [10, lemma 27 in [11] and the fact that a numerical semigroup is irreducible if and only if it is symmetric or pseudo-symmetric, we get the following result.

Lemma 2. Let $S$ be an irreducible numerical semigroup and $x$ a minimal generator of $S$ such that $x<\mathrm{F}(S)$. Then, $\mathrm{F}(S)-x \in \mathrm{PF}(S \backslash\{x\})$ if and only if $2 x-\mathrm{F}(S) \notin S$.

There are many characterizations of symmetric and pseudo-symmetric numerical semigroups. Probably, the most used is the following that appears in 9 .

Lemma 3. Let $S$ be a numerical semigroup. Then:

(1) $S$ is symmetric if $\mathrm{F}(S)$ is odd and if $x \in \mathbb{Z} \backslash S$, then $\mathrm{F}(S)-x \in S$.

(2) $S$ is pseudo-symmetric if $\mathrm{F}(S)$ is even and if $x \in \mathbb{Z} \backslash S$, then either $x=\frac{\mathrm{F}(S)}{2}$ or $\mathrm{F}(S)-x \in S$.

Let $S$ be a numerical semigroup. The cardinal of its set of gaps, $\mathrm{G}(S)=\mathbb{N} \backslash S$, is called the genus of $S$ and it is usually denoted by $\mathrm{g}(S)$.

Lemma 4. Let $S$ be a numerical semigroup. Then:

(1) $S$ is symmetric if and only if $\mathrm{g}(S)=\frac{\mathrm{F}(S)+1}{2}$.

(2) $S$ is pseudo-symmetric if and only if $\mathrm{g}(S)=\frac{\mathrm{F}(S)+2}{2}$.

As a consequence of the above results we have that the set of symmetric (resp. pseudo-symmetric) numerical semigroups is the set of irreducible numerical semigroups with odd (resp. even) Frobenius number, and that a numerical semigroup, $S$, is irreducible if and only if $\mathrm{g}(S)=\left\lceil\frac{\mathrm{F}(S)+1}{2}\right\rceil$ (here $\lceil z\rceil=\min \{n \in \mathbb{Z}: z \leq n\}$ the ceiling part of any rational number $z$ ).

Proposition 5. Let $S$ be an irreducible numerical semigroup with Frobenius number $F$ and let $x$ be a minimal generator of $S$ verifying that:

(1) $x<F$, 
THE TREE OF IRREDUCIBLE NUMERICAL SEMIGROUPS WITH FIXED FROBENIUS NUMBER

(2) $2 x-F \notin S$,

(3) $3 x \neq 2 F$, and

(4) $4 x \neq 3 F$.

Then, $\bar{S}=(S \backslash\{x\}) \cup\{F-x\}$ is an irreducible numerical semigroup with Frobenius number $F$.

Proof. To prove the result it is enough to see that $\bar{S}$ is closed under addition since in that case $\bar{S}$ is a numerical semigroup with Frobenius number $F$ and $\mathrm{g}(\bar{S})=\mathrm{g}(S)$. Hence, by Lemma 4 we get that $\bar{S}$ is irreducible.

The addition of two elements in $S \backslash\{x\}$ is an element in $S \backslash\{x\}$ (Lemma 11). By Lemma 2 we deduce that $F-x \in \operatorname{PF}(S \backslash\{x\})$ and then $F-x+s \in \bar{S}$ for all $s \in S \backslash\{x\}$.

To conclude the proof, we see that $2(F-x) \in S \backslash\{x\}$. For the sake of that we distinguish two cases:

- Assume that $S$ is symmetric. Since $2 x-F \notin S$, then by Lemma 3 we have that $F-(2 x-F) \in S$. Thus, $2(F-x) \in S$. Moreover, since $3 x \neq 2 F$, then $2(F-x) \neq x$ and consequently $2(F-x) \in S \backslash\{x\}$.

- Assume now that $S$ is pseudo-symmetric. Since $2 x-F \notin S$, by Lemma 3 we have that either $2 x-F=\frac{F}{2}$ or $F-(2 x-F) \in S$. Since $4 x \neq 3 F$ then $2 x-F \neq \frac{F}{2}$. Thus, $2(F-x) \in S$. Furthermore, since $3 x \neq 2 F$, then $2(F-x) \neq x$ and consequently $2(F-x) \in S \backslash\{x\}$.

The smallest positive integer belonging to a numerical semigroup $S$ is called the multiplicity of $S$ and it is denoted by $\mathrm{m}(S)$. Note that $\mathrm{m}(S)$ is always a minimal generator of $S$.

Corollary 6. Let $S$ be an irreducible numerical semigroup with $\mathrm{m}(S)<\frac{\mathrm{F}(S)}{2}$. Then, $\bar{S}=(S \backslash\{\mathrm{m}(S)\}) \cup\{\mathrm{F}(S)-\mathrm{m}(S)\}$ is an irreducible numerical semigroup with $\mathrm{F}(\bar{S})=\mathrm{F}(S)$ and $\mathrm{m}(\bar{S})>\mathrm{m}(S)$.

Proof. Let us see that $\mathrm{m}(S)$ is a minimal generator of $S$ that holds the conditions (11)-(14) in the above proposition. Indeed, since $\mathrm{m}(S)<\frac{\mathrm{F}(S)}{2}$ then (11) is clearly satisfied. Furthermore, $2 \mathrm{~m}(S)-\mathrm{F}(S)<0$ and then (2) is also verified. Also, since $\mathrm{m}(S)<\frac{\mathrm{F}(S)}{2}$, (3) and (4) are easily deduced. Finally, $\mathrm{m}(\bar{S})>\mathrm{m}(S)$ because $\bar{S}=(S \backslash\{\mathrm{m}(S)\}) \cup\{\mathrm{F}(S)-\mathrm{m}(S)\}$ and $\mathrm{F}(S)-\mathrm{m}(S)>\mathrm{m}(S)$.

Note that if we take an irreducible numerical semigroup and we apply repeatedly the construction given in Corollary 6, we get, in a finite number of steps, an irreducible numerical semigroup with all its minimal generators larger than half of its Frobenius number. The following result shows that such a semigroup is unique.

Proposition 7. Let $F$ be a positive integer. Then, there exists an unique irreducible numerical semigroup $\mathrm{C}(F)$ with Frobenius number $F$ and all its minimal generators larger than $\frac{F}{2}$. Moreover,

$$
\mathrm{C}(F)=\left\{\begin{aligned}
\left\{0, \frac{F+1}{2}, \rightarrow\right\} \backslash\{F\} & \text { if } F \text { is odd, } \\
\left\{0, \frac{F}{2}+1, \rightarrow\right\} \backslash\{F\} & \text { if } F \text { is even, }
\end{aligned}\right.
$$

(here, if $a_{1}<\cdots<a_{k}$ are integers, then we use $\left\{a_{1}, \ldots, a_{k}, \rightarrow\right\}$ to denote the set $\left\{a_{1}, \ldots, a_{k}\right\} \cup\left\{z \in \mathbb{Z}: z>a_{k}\right\}$.) 
Proof. It is clear that $\mathrm{C}(F)$ is a numerical semigroup with Frobenius number $F$. Furthermore, $\mathrm{g}(\mathrm{C}(F))=\frac{F+1}{2}$ if $F$ is odd and $\mathrm{g}(\mathrm{C}(F))=\frac{F+2}{2}$ if $F$ is even. By applying Lemma 4 we have that $\mathrm{C}(F)$ is irreducible.

Assume now that $S$ is an irreducible numerical semigroup with Frobenius number $F$ and with all its minimal generators larger than $\frac{F}{2}$, then, it is clear that $S \subseteq$ $\mathrm{C}(F)$. By applying now that $S$ is maximal in the set of numerical semigroups with Frobenius number $F$, we conclude that $S=\mathrm{C}(F)$.

For a given $S \in \mathcal{I}(F)$, we define the following sequence of elements in $\mathcal{I}(F)$ :

$$
\begin{aligned}
& S_{0}=S \text {, } \\
& S_{n+1}=\left\{\begin{array}{cc}
\left(S_{n} \backslash\left\{\mathrm{m}\left(S_{n}\right)\right\}\right) \cup\left\{F-\mathrm{m}\left(S_{n}\right)\right\} & \text { if } \mathrm{m}\left(S_{n}\right)<\frac{F}{2}, \\
S_{n} & \text { otherwise. }
\end{array}\right.
\end{aligned}
$$

Let $k=\#\left\{s \in S: s<\frac{F}{2}\right\}-1$ (here \#A stands for the cardinal of the finite set $A)$, then $S_{k+n}=\mathrm{C}(F)$ for all $n \in \mathbb{N}$.

Example 8. Let $S=\langle 4,5\rangle$, which is an irreducible numerical semigroup with Frobenius number 11. Then:

$$
\begin{aligned}
& S_{0}=\langle 4,5\rangle, \\
& S_{1}=(\langle 4,5\rangle \backslash\{4\}) \backslash\{7\}=\langle 5,7,8,9\rangle, \\
& S_{2}=(\langle 5,7,8,9\rangle \backslash\{5\}) \backslash\{6\}=\langle 6,7,8,9,10\rangle=\mathrm{C}(11), \\
& S_{2+n}=\mathrm{C}(11)=\langle 6,7,8,9,10\rangle, \text { for all } n \in \mathbb{N} .
\end{aligned}
$$

Note that in this case $k=\#\left\{s \in S: s<\frac{11}{2}\right\}-1=\#\{0,4,5\}-1=2$.

A (directed) graph $G$ is a pair $(V, E)$ where $V$ is a nonempty set whose elements are called vertices and $E$ is a subset of $\{(v, w) \in V \times V: v \neq w\}$. The elements in $E$ are called edges. A path that connects two vertices of $G, v$ and $w$, is a sequence of distinct edges in the form $\left(v_{0}, v_{1}\right),\left(v_{1}, v_{2}\right), \ldots,\left(v_{n-1}, v_{n}\right)$ with $v_{0}=v$ and $v_{n}=w$ for some $n \in \mathbb{N}$.

A graph $G$ is a tree if there exists a vertex $r$ (called the root of $G$ ) such that for any other vertex of $G, v$, there exists an unique path connecting $v$ and $r$. If $(v, w)$ is a edge of the tree, then, it is said that $v$ is a child of $w$. A leaf is a vertex without children.

Let $\mathcal{I}(F)$ be the set of irreducible numerical semigroups with Frobenius number $F$. We define the directed graph $\mathrm{G}(\mathcal{I}(F))=(V, E)$ as follows:

- $V=\mathcal{I}(F)$, and

- $(T, S) \in E$ if $m(T)<\frac{F}{2}$ and $S=(T \backslash\{\mathrm{m}(T)\}) \cup\{F-\mathrm{m}(T)\}$.

Theorem 9. Let $F$ be a positive integer. The directed graph $\mathrm{G}(\mathcal{I}(F))$ is a tree with root $\mathrm{C}(F)$. Moreover, if $S \in \mathcal{I}(F)$, then, the children of $S$ in $\mathrm{G}(\mathcal{I}(F)$ ) are $\left(S \backslash\left\{x_{1}\right\}\right) \cup\left\{F-x_{1}\right\}, \ldots,\left(S \backslash\left\{x_{r}\right\}\right) \cup\left\{F-x_{r}\right\}$, where $\left\{x_{1}, \ldots, x_{r}\right\}$ is the set of minimal generators, $x$, of $S$ that verify:

(1) $\frac{F}{2}<x<F$,

(2) $2 x-F \notin S$,

(3) $3 x \neq 2 F$,

(4) $4 x \neq 3 F$,

(5) $F-x<\mathrm{m}(S)$.

Proof. As a direct consequence of the construction after Proposition 7 we have that $\mathrm{G}(\mathcal{I}(F))$ is a tree with root $\mathrm{C}(F)$. 
THE TREE OF IRREDUCIBLE NUMERICAL SEMIGROUPS WITH FIXED FROBENIUS NUMBER

(Sufficiency) By Proposition 5 we know that $T=(S \backslash\{x\}) \cup\{F-x\} \in \mathcal{I}(F)$. By condition (5) we have that $\mathrm{m}(T)=F-x$ and by condition (10) that $\mathrm{m}(T)<\frac{F}{2}$. Hence, $S=(T \backslash\{\mathrm{m}(T)\}) \cup\{F-\mathrm{m}(T)\}$, so $T$ is a child of $S$.

(Necessity) Let $T$ be a child of $S$. Then, $\mathrm{m}(T)<\frac{F}{2}$ and $S=(T \backslash\{\mathrm{m}(T)\}) \cup\{F-$ $\mathrm{m}(T)\}$. Then, $T=(S \backslash\{F-\mathrm{m}(T)\}) \cup\{F-(F-\mathrm{m}(T))\}$. To conclude the proof it is enough to see that $F-\mathrm{m}(T)$ is a minimal generator if $S$ verifying conditions (11)-(5):

(1) Since $F-\mathrm{m}(T) \notin T$ and $S=(T \backslash\{\mathrm{m}(T)\}) \cup\{F-\mathrm{m}(T)\}$, we easy deduce that $F-\mathrm{m}(T)$ is a minimal generator of $S$. Furthermore, since $\mathrm{m}(T)<\frac{F}{2}$ then $\frac{F}{2}<F-\mathrm{m}(T)<F$.

(2) If $2(F-\mathrm{m}(T))-F \in S$, we have that $F-2 \mathrm{~m}(T) \in S$. However, $2 \mathrm{~m}(T) \in S$ since $2 \mathrm{~m}(T) \in T \backslash\{\mathrm{m}(T)\}$, we have that $F=F-2 \mathrm{~m}(T)+2 \mathrm{~m}(T) \in S$ which is not possible.

(3) If $3(F-\mathrm{m}(T))=2 F$ then $F=3 \mathrm{~m}(T) \in S$ contradicting the definition of Frobenius number.

(4) If $4(F-\mathrm{m}(T))=3 F$ then $F=4 \mathrm{~m}(T) \in S$ which is not possible.

(5) Since $\mathrm{m}(T)<\frac{F}{2}$ then $\mathrm{m}(T)<F-\mathrm{m}(T)$ and then, $\mathrm{m}(T)<\mathrm{m}(S)$. Thus, $F-(F-\mathrm{m}(T))<\mathrm{m}(S)$.

We conclude this section by illustrating the applicability of the above result to construct, explicitly, the tree $\mathrm{G}(\mathcal{I}(F))$.

Example 10. Let us compute the whole set of irreducible numerical semigroups with Frobenius number 11. We start by the root of the tree, $\mathrm{C}(11)=\langle 6,7,8,9,10\rangle$. The minimal generators that hold the conditions (1)-(5) are: $x_{1}=8, x_{2}=7$ and $x_{3}=6$. Then, the children of $\mathrm{C}(11)$ are:

$$
\begin{aligned}
& (\langle 6,7,8,9,10\rangle \backslash\{8\}) \cup\{3\}=\langle 3,7\rangle \\
& (\langle 6,7,8,9,10\rangle \backslash\{7\}) \cup\{4\}=\langle 4,6,9\rangle \\
& (\langle 6,7,8,9,10\rangle \backslash\{6\}) \cup\{5\}=\langle 5,7,8,9\rangle
\end{aligned}
$$

Thus, the tree $\mathrm{G}(\mathcal{I}(11))$ starts in the form:

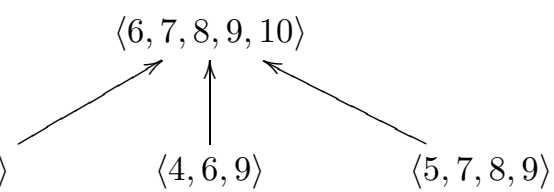

Next, we compute the children of $\langle 3,7\rangle,\langle 4,6,9\rangle$ and $\langle 5,7,8,9\rangle$. First, we observe that $\langle 3,7\rangle$ has no children. The unique generator of $\langle 4,6,9\rangle$ with the conditions of Theorem 9 is $x_{1}=9$. Then, $\langle 4,6,9\rangle$ only has a child, which is $(\langle 4,6,9\rangle \backslash\{9\}) \cup\{2\}=$ $\langle 2,13\rangle$. For $\langle 5,7,8,9\rangle$, only $x_{1}=7$ holds the conditions of Theorem [9, so the unique 
child of $\langle 5,7,8,9\rangle$ is $(\langle 5,7,8,9\rangle \backslash\{7\}) \cup\{4\}=\langle 4,5\rangle$. Hence, the tree continues as:

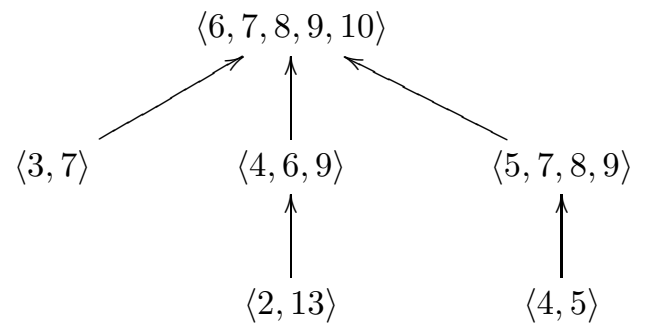

Finally, since $\langle 2,3\rangle$ and $\langle 4,5\rangle$ do not have minimal generators with the conditions of Theorem 9, they have no children. Thus, the tree $\mathrm{G}(\mathcal{I}(11))$ is completed.

\section{A Kunz-Coordinates vector construction of the tree $\mathrm{G}(\mathcal{I}(F))$}

In this section we use a different encoding of a numerical semigroups to compute the tree $\mathrm{G}(\mathcal{I}(F))$ for any positive integer $F$. We present an analogous construction to the one in the section above but based on manipulating vectors in $\{0,1\}^{F}$. It leads us to an efficient procedure to compute the set of irreducible numerical semigroups with Frobenius number $F$.

Let $S$ be a numerical semigroup and $n \in S$. The Apéry set of $S$ with respect to $n$ is the set $\operatorname{Ap}(S, n)=\{s \in S: s-n \notin S\}$. This set was introduced by Apéry in [1.

The following characterization of the Apéry set that appears in [9] will be useful for our development.

Lemma 11. Let $S$ be a numerical semigroup and $n \in S \backslash\{0\}$. Then $\operatorname{Ap}(S, n)=$ $\left\{0=w_{0}, w_{1}, \ldots, w_{n-1}\right\}$, where $w_{i}$ is the least element in $S$ congruent with $i$ modulo $n$, for $i=1, \ldots, n-1$.

Moreover, the set $\operatorname{Ap}(S, n)$ completely determines $S$, since $S=\langle\operatorname{Ap}(S, n) \cup$ $\{n\}\rangle$ (see [7]), and then, we can identify $S$ with its Apéry set with respect to $n$. The set $\operatorname{Ap}(S, n)$ contains, in general, more information than an arbitrary system of generators of $S$. For instance, Selmer in 12 gives the formulas, $\mathrm{g}(S)=$ $\frac{1}{n}\left(\sum_{w \in \operatorname{Ap}(S, n)} w\right)-\frac{n-1}{2}$ and $\mathrm{F}(S)=\max (\operatorname{Ap}(S, n))-n$. One can also test if a nonnegative integer $s$ belongs to $S$ by checking if $w_{s}(\bmod m) \leq s$.

We consider an useful modification of the Apéry set that we call the Kunzcoordinates vector as in 3 . Let $S$ be a numerical semigroup and $n \in S \backslash\{0\}$. By Lemma 11, $\operatorname{Ap}(S, n)=\left\{w_{0}=0, w_{1}, \ldots, w_{n-1}\right\}$, with $w_{i}$ congruent with $i$ modulo $n$. The Kunz-coordinates vector of $S$ with respect to $n$ is the vector $\mathcal{K}(S, n)=x \in \mathbb{N}^{n-1}$ with components $x_{i}=\frac{w_{i}-i}{n}$ for $i=1, \ldots, n-1$. We say that $x \in \mathbb{N}^{n-1}$ is a Kunzcoordinates vector with respect to $n$ if there exists a numerical semigroup whose Kunz-coordinates vector is $x$. If $x \in \mathbb{N}^{n-1}$ is a Kunz-coordinates vector, we denote by $S_{x}$ the numerical semigroup such that $\mathcal{K}\left(S_{x}, n\right)=x$.

The Kunz-coordinates vectors were introduced in [5] and have been previously analyzed when $n=\mathrm{m}(S)$ in $[3,7$.

In the following result, whose proof is immediate from the results in the section above, it is shown how to get the multiplicity and the genus of a numerical semigroup with Frobenius number $F$ from its Kunz-coordinates vector with respect to $F+1$.

Lemma 12. Let $S$ be a numerical semigroup and $x=\mathcal{K}(S, \mathrm{~F}(S)+1) \in \mathbb{N}^{F}$. Then: 
THE TREE OF IRREDUCIBLE NUMERICAL SEMIGROUPS WITH FIXED FROBENIUS NUMBER

(1) $x \in\{0,1\}^{\mathrm{F}(S)}$,

(2) $\mathrm{G}(S)=\left\{i \in\{1, \ldots, \mathrm{F}(S)\}: x_{i}=1\right\}$,

(3) $\mathrm{m}(S)=\left\{\begin{array}{cl}\min \left\{i: x_{i}=0\right\} & \text { if }\left\{i: x_{i}=0\right\} \neq \emptyset, \\ \mathrm{F}(S)+1 & \text { otherwise }\end{array}\right.$,

(4) $\mathrm{g}(S)=\sum_{i=1}^{F} x_{i}$

We denote by $\operatorname{SG}(S)=\{h \in \mathbb{N} \backslash S: S \cup\{h\}$ is a numerical semigroup $\}$ the set of special gaps of the numerical semigroup $S$. It is well-known (see 9]) that $S$ is irreducible if and only if \# $\mathrm{SG}(S)=1$. Furthermore, $\mathrm{SG}(S)$ can be computed from $x=\mathcal{K}(S, \mathrm{~F}(S)+1)$ by applying the characterization of $\mathrm{SG}(S)$ in terms of the Apéry set that appears in [6]:

$$
\begin{aligned}
\mathrm{SG}(S)=\{i \in\{1, \ldots, \mathrm{F}(S)\}: & x_{i}=1, \\
& x_{j} \geq x_{i+j} \quad, \text { for all } j=i, \ldots, \mathrm{F}(S)-i, \text { and } \\
& \left.x_{2 i}=0 \text { if } i<\frac{\mathrm{F}(S)}{2}\right\}
\end{aligned}
$$

The construction of the tree of irreducible numerical semigroups with fixed Frobenius number in the section above consists of adding and removing certain elements to the semigroups to get the child of an irreducible numerical semigroup (Theorem 9). The following lemma, whose proof is trivial, informs us about the translations of adding and removing elements of a numerical semigroups in its Kunz-coordinates vector.

Lemma 13. Let $S$ be a numerical semigroup and $\mathrm{e}_{i}$ the $\mathrm{F}(S)$-tuple having a 1 as its ith entry and zeros otherwise, for $i=1, \ldots, \mathrm{F}(S)$. Then:

(1) If $h \in \mathrm{SG}(S) \backslash\{\mathrm{F}(S)\}$, then $\mathcal{K}(S \cup\{h\}, \mathrm{F}(S)+1)=\mathcal{K}(S, \mathrm{~F}(S)+1)-\mathrm{e}_{h}$.

(2) If $n<\mathrm{F}(S)+1$ is a minimal generator of $S$, then $\mathcal{K}(S \backslash\{n\}, \mathrm{F}(S)+1)=$ $\mathcal{K}(S, \mathrm{~F}(S)+1)+\mathrm{e}_{n}$.

In the section above we proved that there is a numerical semigroup of special importance for the construction of the tree $\mathrm{G}(\mathcal{I}(F)), \mathrm{C}(F)$. The following result shows the Kunz-coordinates vector of this semigroup with respect to $F+1$.

Lemma 14. Let $F$ be a positive integer. Then, $\mathcal{K}(\mathrm{C}(F), F+1)=(\overbrace{1, \ldots, 1}^{\left\lceil\frac{F+1}{2}\right\rceil-1}, \overbrace{0, \ldots, 0}^{F-\left\lceil\frac{F+1}{2}\right\rceil}, 1) \in$ $\mathbb{N}^{F}$.

For any positive integer $F$, we define the directed graph $\mathrm{G}^{K}(\mathcal{I}(F))$ as the translation of $\mathrm{G}(\mathcal{I}(F))$ in terms of the Kunz-coordinates vectors with respect to $F+1$. The vertices of $\mathrm{G}^{K}(\mathcal{I}(F))$ is the set $\{\mathcal{K}(S, F+1): S \in \mathcal{I}(F)\}$, and $(x, y)$ is an edge of $\mathrm{G}^{K}(\mathcal{I}(F))$ if $m=\min \left\{i: x_{i}=0\right\}<\frac{F}{2}$ and $y=x+\mathrm{e}_{m}-\mathrm{e}_{F-m}$ (Lemma 13).

Now, we proceed to translate the conditions of Theorem 9 in terms of the Kunzcoordinates vector.

Theorem 15. Let $F$ be a positive integer. The directed graph $\mathrm{G}^{K}(\mathcal{I}(F))$ is a tree with root $\hat{x}=(\overbrace{1, \ldots, 1}^{\left\lceil\frac{F+1}{2}\right\rceil-1}, \overbrace{0, \ldots, 0}^{F-\left\lceil\frac{F+1}{2}\right\rceil}, 1)$. Moreover, if $x$ is the Kunz-coordinates vector with respect to $F$ of an irreducible numerical semigroup with Frobenius number $F$, then, the children of $x$ in $\mathrm{G}^{K}(\mathcal{I}(F))$ are $x+\mathrm{e}_{n_{1}}-\mathrm{e}_{F-n_{1}}, \ldots, x+\mathrm{e}_{n_{r}}-\mathrm{e}_{F-n_{r}}$, where $\left\{n_{1}, \ldots, n_{r}\right\}$ verifies: 
(1) $x_{n_{i}}=0$, for all $i=1, \ldots, r$,

(2) $x_{k}+x_{j} \geq 1$ if $k+j=n_{i}$, for all $i=1, \ldots, r$,

(3) $\frac{F}{2}<n_{i}<F$, for all $i=1, \ldots, r$,

(4) $x_{2 n_{i}-F}=1$, for all $i=1, \ldots, r$,

(5) $3 n_{i} \neq 2 F$, for all $i=1, \ldots, r$,

(6) $4 n_{i} \neq 3 F$, for all $i=1, \ldots, r$,

(7) $n_{i}>F-\min \left\{j: x_{j}=0\right\}$.

Proof. Note that conditions (1) and (2) are equivalent to say that $n_{i}$ is a minimal generator smaller than $F$ of $S_{x}$.

Items $(3)-(7)$ are the translations of conditions (1) - (15) in Theorem 9 ,

Note that the satisfaction of conditions (1) - (7) in Theorem [15 is checked by analyzing only the connections between the $F$ components of a $0-1$ vector. From a computational viewpoint this is much easier than checking conditions (11)-(5) in Theorem 9. Observe also that $x+\mathrm{e}_{n}-e_{F-n}$ in our case $\left(x \in\{0,1\}^{F}, n\right.$ a minimal generator and $F-n$ a special gap of $S_{x}$ ) consists of swapping $x_{n}$ with $x_{F-n}$. Hence, the computational effort is small at each step.

Other advantage of this approach is that in general, the computation of the Apéry sets and the Kunz-coordinates vector of a numerical semigroup is hard. However, by the above theorem we do not need to compute the Kunz-coordinates vectors of the semigroups appearing in the tree $\mathrm{G}^{K}(\mathcal{I}(F))$ since by starting with $\left\lceil\frac{F+1}{2}\right\rceil-1 \quad F-\left\lceil\frac{F+1}{2}\right\rceil$

$(\overbrace{1, \ldots, 1}, \overbrace{0, \ldots, 0}, 1)$ and manipulating adequately the $0-1$ vectors representing these semigroups we are assured to get Kunz-coordinates vectors of irreducible numerical semigroups with Frobenius number $F$.

A vertex in $\mathrm{G}^{K}(\mathcal{I})$ ) has at most $\frac{F}{2}$ children (the number of possible swappings when the parent has $\frac{F}{2}$ ones and $\frac{F}{2}$ zeroes). Then, the complexity of each step (building the children of a vertex) has computational complexity $O\left(F^{2} \delta(F)\right)$ where $\delta(F)$ is the computational complexity of checking the conditions in Theorem 15] which is clearly a polynomial in $F$. Also, the height of the tree is bounded above by $\frac{F}{2}$. Hence, computing the tree $\mathrm{G}^{K}(\mathcal{I}(F))$ is polynomial-time doable.

Note that the tree structure of the set of irreducible numerical semigroups with a fixed Frobenius number is useful when one is interested in finding irreducible numerical semigroups with some extra properties. For instance, this representation is useful when searching for the set of irreducible oversemigroups of a given numerical semigroup with a fixed Frobenius number or those that also has a fixed multiplicity (see [3]).

The following example illustrates the construction of the tree $\mathrm{G}^{K}(\mathcal{I}(F))$ in the same case than in Example10.

Example 16. Let us compute the whole set of irreducible numerical semigroups with Frobenius number $F=11$ by using Kunz-coordinates. In this case $\left\lceil\frac{F+1}{2}\right\rceil=$ 6. First we star with $x=(1,1,1,1,1,0,0,0,0,0,1)$ which is the root of the tree $\mathrm{G}^{K}(\mathcal{I}(11))$. The set of indices verifying conditions of Theorem[15 is $\{6,7,8\}$. Note that to get this set, first we look for the components in $x$ that are equal to zero (Condition (1)), which are $\{6,7,8,9,10\}$; then we check for those elements that verify conditions (3) and (7) (no elements are removed in this step). From those we filter by those that verify Condition (4) (9 and 10 are removed from the set since $\left.x_{7}=x_{9}=0\right)$. Finally we check for the rest of conditions, which are in 
this case verified by all the remainder elements. Therefore, the children of $x$ are $x^{1}=x+\mathrm{e}_{8}-\mathrm{e}_{3}, x^{2}=x+\mathrm{e}_{7}-\mathrm{e}_{4}$ and $x^{3}=x+\mathrm{e}_{6}-\mathrm{e}_{5}$. Then, the first part of the tree is:

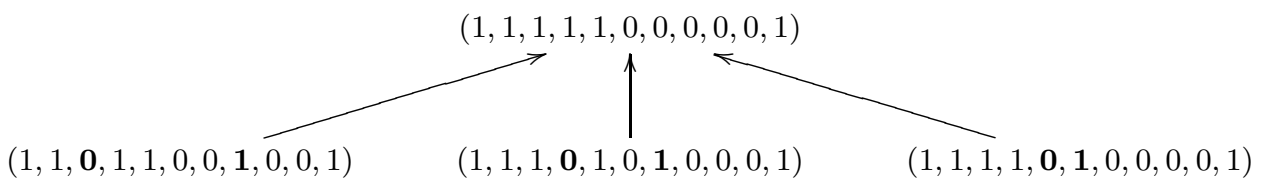

The elements which are swapped to get each child are marked in boldface.

Now, we compute the children of $x^{1}, x^{2}$ and $x^{3}$. By an analogous procedure we get that $x^{1}$ has no children, for $x^{2}$ the unique index verifying the conditions of Theorem 15 is $n_{1}=9$, being the unique child $x^{4}=x^{2}+\mathrm{e}_{9}-\mathrm{e}_{2}$. Also, $x^{3}$ has only a child $x^{5}=x^{3}+\mathrm{e}_{7}-\mathrm{e}_{4}$ which comes from the unique index that verifies the conditions, $n_{1}=7$. Then, the continuation of the tree is:

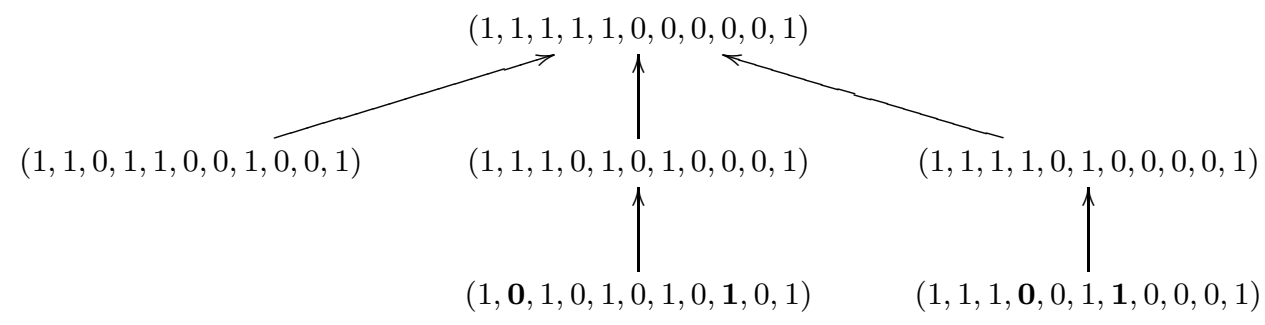

Finally, we see that $x^{4}$ and $x^{5}$ have no children, so the tree $\mathrm{G}^{K}(\mathcal{I}(11))$ is completed.

\section{REFERENCES}

[1] Apéry, R. (1946). Sur les branches superlinéaires des courbes algébriques. C. R. Acad. Sci. Paris 222, 1198-2000.

[2] Barucci, V., Dobbs, D.E., and Fontana, M. (1997). Maximality properties in numerical semigroups and applications to one-dimensional analitically irreducible local domains. Memoirs of the American Mathematical Society. Vol.125, n.598.

[3] Blanco, V. and Puerto, J. (2011). Integer programming for decomposing numerical semigroups into m-irreducible numerical semigroups. Submitted. Available in http://arxiv.org/abs/1101.4112.

[4] Fröberg, R., Gottlieb, C., and Häggkvist, R. On numerical semigroups, Semigroup Forum 35 (1987) 6383.

[5] Kunz, E. (1987). Über dir Klassifikation numerischer Halbgruppen, Regensburger matematische schriften 11.

[6] Rosales, J.C, and Branco, M.B. (2002). Numerical semigroups that can be expressed as an intersection of symmetric numerical semigroups. J. Pure Appl. Algebra 171 (2-3) (2002), 303-314.

[7] Rosales, J. C., García-Sánchez, P. A., García-García, J. I., and Branco, M. B. (2002). Systems of inequalities and numerical semigroups. J. London Math. Soc. (2) 65, no. 3, 611-623.

[8] Rosales, J.C, and Branco, M.B. (2003). Irreducible numerical semigroups, Pacific J. Math. 209 (2003), 131-143.

[9] Rosales, J.C. and García-Sanchez, P.A. (2009). Numerical semigroups, Springer, New York, NY, 2009. ISBN: 978-1-4419-0159-0.

[10] Rosales, J.C. (2008). Numerical semigroups that differ from a symmetric numerical semigroup in one element, Algebra Colloquium 15 (2008), 23-32

[11] Rosales, J.C. (2006). Adding or removing an element from a peseudo-symmetric numerical semigroup, Boll. Unione Mat. Ital. Sez. B Art. Ric. Matem. (8) 9 (2006), 681-696.

[12] Selmer, E.S. (1977). On a linear Diophantine problem of Frobenius, J. Reine Angew. Math. 293/294, 1-17. 
Departamento de Álgebra, Universidad de Granada 